\title{
Power factor control using brushless doubly fed reluctance machines
}

\author{
M.G.Jovanović ${ }^{1}$ and R.E.Betz ${ }^{2}$ \\ ${ }^{1}$ University of Northumbria at Newcastle \\ School of Engineering, Newcastle upon Tyne NE1 8ST, UK \\ Fax: +44-191-2273684;E-mail: milutin.jovanovic@unn.ac.uk \\ ${ }^{2}$ University of Newcastle \\ Department of Electrical and Computer Engineering, NSW 2308, Australia \\ Fax: +61-2-49216993; E-mail: reb@ecemail.newcastle.edu.au
}

\begin{abstract}
The brushless doubly-fed machine (BDFM) allows the use of a partially rated inverter in many variable speed applications with limited speed variations. In its induction machine form (BDFIM), however, the BDFM has significant rotor losses and poor efficiency due to the cage rotor design. A reluctance version of the BDFM, the brushless doubly-fed reluctance machine (BDFRM), ideally has no rotor losses, and therefore offers the potential for greater efficiency and simpler control compared to the BDFIM. A theoretical study of this interesting machine is very important in order to gain a thorough understanding of its unusual operation, control features and compromises between optimal performance and the size of the inverter and the machine. This paper will specifically concentrate on power factor control properties of the machine and trade-offs with other control objectives such as maximum torque per inverter ampere and minimum copper losses. To date this kind of analysis has not appeared in the literature.
\end{abstract}

\section{INTRODUCTION}

$\mathrm{W}$ ITH the advent of power electronics and microprocessor technology, it has become possible to better control and utilise brushless doubly fed machines (BDFMs) to the point where they have started to be considered as a potential alternative to the existing drive solutions in a variety of low to medium performance industrial applications. The main reasons for this growing interest can be summarised as follows:

- The feeding converter only has to handle the slip power, which means that significant cost savings (up to $30 \%$ ) can be made compared to conventional systems with fully rated inverters [1]. This is particularly true in larger drives with limited variable speed ranges (pumps [2], compressors, wind turbines $[3,4]$, heating, ventilation and air-conditioning etc.) where, despite the falling market prices, the power hardware still represents a major portion of the capital cost. A cost penalty would incurred for the machine but this is more than offset by the reduction in cost of the feeding converter. Another benefit of smaller inverter rating is the improved supply quality, and consequent lower filtering requirement, as less harmonics are injected into the grid.

- The reliability of brushless design and consequent lower maintenance make BDFMs superior to doubly fed wound rotor induction machines in slip energy recovery systems (static Kramer or Scherbius cascades).

- The BDFM shares all the advantages of doubly fed machines over singly excited counterparts - operational mode flexibility, the greater control freedom and the possibility of being operated at sub-synchronous and super-synchronous speeds in both motoring and generating regimes. It can operate as a standard or doubly excited induction machine and as a fixed or adjustable speed synchronous turbo-machine. The latter operating mode means that high speed, field weakened traction applications [5], as well as high frequency generators [6] become feasible. In the former applications, the ability of the BDFM to function as an induction machine in case of inverter failure is an important "fail-safe" feature. From a control viewpoint, one merit of the machine is that one can not only control the real power from the machine, but also the power factor or efficiency.

The use of a cageless synchronous reluctance machine (Syncrel) rotor (Fig. 1), instead of a special cage one, further improves the BDFM's overall performance (this is known as the brushless doubly fed reluctance machine, or BDFRM). The machine should be more efficient and mechanically robust, as well as much simpler to control. Furthermore, the BDFRM does not suffer from stability problems inherent with the BDFIM around the zero-torque operating point at synchronous speed of the grid-connected winding field [7]. This means that, unlike the BDFIM, the BDFRM can be operated stably over the entire speed range down to standstill. Therefore, this paper will focus on the BDFRM.

As can be seen from Fig. 1, the machine has two separate stator windings of different pole numbers and a rotor with the pole-number (or nests with the BDFIM) being half the sum of the stator poles for torque to be produced. Note that this implies that it is possible for the rotor to have an odd pole number (for 4/2-pole stator windings) in contrast to a conventional machine where the pole number is always even. The reluctance rotor may have any of the standard Syncrel forms but, as with the Syncrel, the best performance can be achieved using an axiallylaminated construction shown in the figure [8].

One of the BDFRM's main attributes is its power factor control capability. The power factor in the inverter-fed (secondary) winding is relevant to the inverter size, but is irrelevant to the outside utility network (since the inverter effectively isolates the secondary from the mains supply). However the power factor of the machine's grid connected (primary) winding is of great importance (especially in weak networks) in the light of new regulations coming into force around the world on this issue. To minimise the total current loading (and thus losses) for 


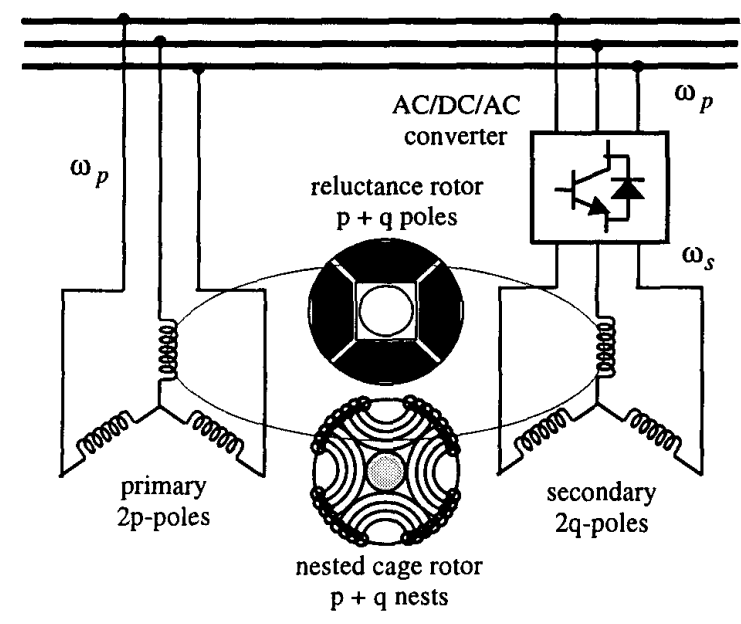

Fig. 1. Structural diagram of the $6 / 2$-pole BDFM with an axially-laminated rotor (top) and nested cage rotor (bottom)

a given real power demand, it is therefore desirable to keep the primary power factor as close as possible to unity. One can also generate VARs into the power grid in order to provide voltage support. However, the price to pay for this is an increased inverter rating and the need for a bidirectional power converter.

The main objective of this paper is to examine maximum power factor control strategies for the BDFRM and trade-offs between them. A comparative study with the maximum torque per inverter ampere and minimum copper loss control properties shall be also carried out where appropriate. Issues related to the influence of the winding pole-numbers on the machine's copper losses shall be also addressed. The material to be presented is an extension to the previous work of the authors on BDFRM control aspects $[9,10]$, and its content is original and is not available in the literature hitherto published on the subject.

\section{Performance Considerations}

This section is concerned with the development and analysis of expressions for an ideal BDFRM (no saturation and iron losses) which are suitable for control implementation. The starting point in this discussion is the space-vector model $^{1}$ for the BDFRM in rotating reference frames $[9,14,15]$ :

$$
\begin{aligned}
& \underline{v}_{p}=R_{p} \underline{i}_{p}+\frac{d \underline{\lambda}_{p}}{d t}+j \omega \underline{\lambda}_{p} \\
& \underline{v}_{s}=R_{s} \underline{i}_{s}+\frac{d \underline{\lambda}_{s}}{d t}+j\left(\omega_{r}-\omega\right) \underline{\lambda}_{s} \\
& \underline{\lambda}_{p}=L_{p} \underline{i}_{p}+L_{p s} \underline{i}_{s p}^{*} \\
& \underline{\lambda}_{s}=L_{s} \underline{\underline{i}}_{s}+L_{p s} \underline{i}_{p s}^{*}
\end{aligned}
$$

where subscripts ' $p$ ' and ' $s$ ' denote the primary and secondary windings, $L_{p, s, p s}$ are the constant three phase inductances of the windings (see Appendix A), $\omega_{r}=\omega_{p}+\omega_{s}$ is the rotor

${ }^{1}$ This model is simpler and has fewer parameter compared to that for the BDFIM [11]. This results from the fact that the BDFIM has an additional winding on the rotor. Consequently, the BDFRM is considerably easier to control $[12,13]$

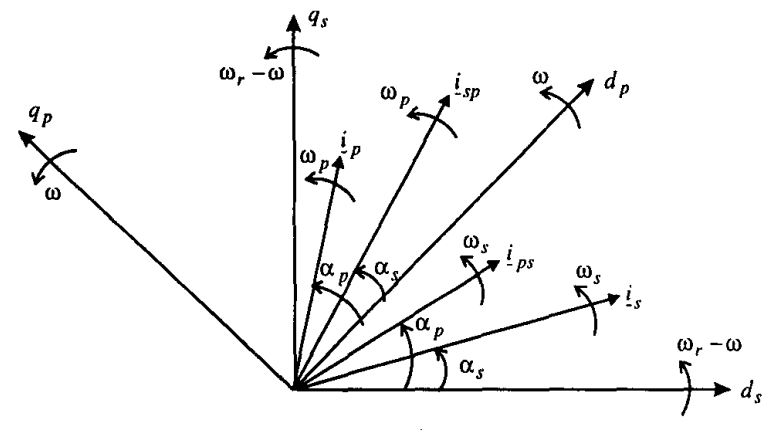

Fig. 2. The reference frames and current angle definitions for the BDFRM

'electrical' velocity ${ }^{2}(\mathrm{rad} / \mathrm{s})$ and $\omega_{p, s}$ are the winding applied frequencies $(\mathrm{rad} / \mathrm{s})$.

Remark 1: Note that the above primary and secondary equations are in two different reference frames, rotating at $\omega$ and $\omega_{r}-\omega$ respectively (Fig. 2), because $\omega_{p} \neq \omega_{s}$. It is interesting that, except for this double frame referencing, the equations are virtually identical in form to those for a double fed wound rotor induction machine, despite the fundamentally different operating principle. Therefore, similar control algorithms can be used for both the machines. $[17,18]$.

Remark 2: The $\underline{i}_{s p}^{*}$ and $\underline{i}_{p s}^{*}$ terms in (3) and (4) are the complex conjugates of the 'coupled' current vectors from the secondary to the primary winding and vice versa and rotate at $\omega_{p}$ and $\omega_{s}$ relative to a stationary frame respectively (Fig. 2). In other words, they represent the $\underline{i}_{s, p}$ vectors referred to their complementary winding side but in a frequency (not turns ratio) sense. This frequency transformation is a consequence of the rotor's modulating influence on the stator mmf waveforms which is the basic principle behind electromechanical energy conversion in the BDFRM $[15,19]$.

Remark 3: As $\underline{i}_{s p}=\underline{i}_{s}$ in their respective frames (Fig. 2) the $d_{p} q_{p}$ components of $\underline{i}_{s p}^{*}$ (and hence the secondary to primary flux coupling term $L_{p s} \underline{i}_{s p}^{*}$ ) can be effectively controlled by the secondary $d_{s} q_{s}$ currents. This forms the basis of field oriented control for this machine, since one only needs to know the secondary frame position [13].

The above equations can be significantly simplified (especially the primary ones) by choosing the generic reference frame $d_{p} q_{p}$ to be aligned with the primary flux vector $\left(\underline{\lambda}_{p}\right)$. This is a natural choice as the primary winding is grid connected and therefore is of fixed line frequency and approximately constant flux magnitude (as $R_{p} i_{p}<<\omega_{p} \lambda_{p}$ ). Doing so, causes (1) and (3) to be with respect to the $\omega=\omega_{p}$ frame whilst (2) and (4) are in the $\omega_{r}-\omega_{p}=\omega_{s}$ frame. The constant flux and frame alignment conditions immediately follow from (3) and can be expressed as:

$$
\lambda_{p}=L_{p} i_{p d}+L_{p s} i_{s d}+j(\underbrace{L_{p} i_{p q}-L_{p s} i_{s q}}_{=0})
$$

Using the general expression for complex power input $P_{3 \phi}+j Q_{3 \phi}=\frac{3}{2}\left(\underline{v}_{p} \underline{i}_{p}^{*}+\underline{v}_{s} \underline{i}_{s}^{*}\right)$, and substituting for (1)-(5),

${ }^{2}$ It can be shown $[14,15]$ that the machine produces usable torque if $\omega_{r}=$ $p_{r} \omega_{r m}=\omega_{p}+\omega_{s}$ where the number of rotor poles is $p_{r}=p+q$ and $p$ and $q(p \neq q)$ are the windings pole-pairs (Fig. 1). The same velocity relationship holds true for the BDFM's ancestor - the cascaded induction machine [16]. 
one can derive, after considerable manipulation, the vector control form expressions ${ }^{3}$ for various performance measures for the machine:

$$
\begin{aligned}
P_{p_{3 \phi}}= & P_{c u_{p}}+P_{p}=\frac{3}{2} R_{p} i_{p}^{2}+\frac{\omega_{p}}{p_{r}} T_{e} \\
P_{s_{3 \phi}}= & P_{c u_{s}}+P_{s}=\frac{3}{2} R_{s} i_{s}^{2}+\frac{\omega_{s}}{p_{r}} T_{e} \\
T_{e}= & \frac{P_{p}+P_{s}}{\omega_{r m}}=p_{r} \frac{P_{p}+P_{s}}{\omega_{p}+\omega_{s}}=\frac{3}{2} p_{r} \frac{L_{p s}}{L_{p}} \lambda_{p} i_{s q} \\
Q_{p}= & \frac{3}{2} \frac{\omega_{p} \lambda_{p}}{L_{p}}\left(\lambda_{p}-L_{p s} i_{s d}\right) \\
Q_{s}= & \frac{3}{2} \omega_{s} \frac{L_{p s}}{L_{p}}\left[\left(\frac{1}{k_{p s}^{2}}-1\right) L_{p s} i_{s}^{2}+\lambda_{p} i_{s d}\right] \\
i_{p}= & \frac{\lambda_{p} \sin \alpha_{s}}{L_{p} \sin \left(\alpha_{p}+\alpha_{s}\right)} ; \quad i_{s}=\frac{\lambda_{p} \sin \alpha_{p}}{L_{p s} \sin \left(\alpha_{p}+\alpha_{s}\right)} \\
P_{c u}= & P_{c u_{p}}+P_{c u_{s}}=\frac{3}{2} \frac{R_{p}}{L_{p}^{2}} \lambda_{p}^{2}+\frac{3}{2}\left(R_{p} \frac{L_{p s}^{2}}{L_{p}^{2}}+R_{s}\right) i_{s q}^{2} \\
& +\frac{3}{2}\left(R_{p} \frac{L_{p s}^{2}}{L_{p}^{2}}+R_{s}\right) i_{s d}^{2}-3 \frac{R_{p}}{L_{p}^{2}} \lambda_{p} L_{p s} i_{s d}
\end{aligned}
$$

where $p_{r}$ is the number of rotor poles, $k_{p s}=L_{p s} / \sqrt{L_{p} L_{s}}$ is the coupling coefficient between the windings and $\alpha_{p, s}$ are the primary and secondary current angles (Fig. 2). The latter are mutually dependent so that (5) is always satisfied. The relationship between these angles in a normalised form can be found in Table I of the Appendix B.

A number of important remarks can be made from the above expressions (and their normalised equivalents in Table I).

Remark 4: The equation (8) shows that one can control the machine torque in an independent manner by the $q$-axis secondary current as $\lambda_{p}=$ const. Since $i_{s q}=i_{s} \sin \alpha_{s}$ (Fig. 2) the maximum torque per inverter ampere (MTPIA) ${ }^{4}$ i.e. the minimum inverter current for a given torque is achieved if the secondary current angle is $\alpha_{s_{\text {mtpia }}}=\pi / 2$. The same equation also indicates that the torque per ampere improves with increasing the $L_{p s} / L_{p}$ ratio as this allows better magnetic coupling between the windings. The ratio of the effective turns per pole of the windings $\left(n_{s} / n_{p}\right)$ should be therefore made as higher as possible as follows from (16) in Appendix A. ${ }^{5}$

Remark 5: As we can see from the primary power expressions (6) and (9), even though we cannot vary the machine primary flux, we are able to regulate the grid power factor in a decoupled fashion since the reactive power $\left(Q_{p}\right)$ is only affected by the $d$-axis secondary current $\left(i_{s d}\right)$. The unity primary

\footnotetext{
${ }^{3}$ The equations are in terms of the secondary currents (directly controllable via the inverter) and the electromagnetic torque which normally appears as an output of a speed control loop. A motoring convention has been adopted as a reference.

${ }^{4}$ The term 'maximum torque per secondary ampere' (MTPSA) will be also interchangeably used throughout the paper for this control strategy.

${ }^{5}$ It has been shown in [10] that with $n_{s}=n_{p}$, the same amount of active material and the same copper losses the BDFRM has lower MTPIA compared to an equivalent Syncrel. However, it has been also demonstrated that the BD. FRM is capable of reaching much higher speeds and developing more power using the same inverter than the Syncrel. Its maximum power output per inverter ampere is consequently better.
}

power factor (UPPF) is obtained when $Q_{p}=0$ and occurs at:-

$$
\alpha_{s_{u p p f}}=\cos ^{-1} \frac{\lambda_{p}}{L_{p s} i_{s}}=\tan ^{-1} \frac{T_{n}}{2}
$$

where $T_{n}$ is the normalised torque output. From (5) and its normalised counterpart in Table $I$, the corresponding primary current angle is $\alpha_{p_{u p p}}=\pi / 2$ as $i_{p d_{u p p f}}=0$. This result is expected as the primary current vector in quadrature with the flux producing $d_{p}$-axis contributes no flux in the machine. The secondary winding would be carrying all of the magnetising current for the machine in this case and the inverter current rating required would consequently be higher.

Remark 6: Following on from the previous remark and setting $Q_{s}=0$ (i.e. $Q_{s n}=\phi_{s}=0$ in the respective expressions of Table I), it is not difficult to show that the unity secondary power factor (USPF) i.e. the lowest inverter VA for a lossless machine, can be accomplished if: ${ }^{6}$

$$
\begin{gathered}
i_{s d_{u s p f}}=-\frac{\lambda_{p}+\sqrt{\lambda_{p}^{2}-4\left(\frac{1}{k_{p s}^{2}}-1\right)^{2} L_{p s}^{2} i_{s q}^{2}}}{2\left(\frac{1}{k_{p s}^{2}}-1\right) L_{p s}}<0 \\
\alpha_{s_{u s p f}}=\tan ^{-1} \frac{-\sqrt{1-T_{n}^{2}\left(\frac{1}{k_{p s}^{2}}-1\right)^{2}}-1}{T_{n}\left(\frac{1}{k_{p s}^{2}}-1\right)}>\frac{\pi}{2}
\end{gathered}
$$

where $i_{s q}$ is directly related to torque (refer to (8)). The significance of good magnetic coupling between the windings is more than evident from the same equations - the higher $k_{p s}$ the lower reactive power $\left(Q_{s n}\right)$ and the better power factor $\left(\cos \phi_{s}\right)$. Unfortunately, the BDFRM is not the best machine in this sense. Due to a highly unusual operating mechanism where the flux side bands are the torque producing components, the main fundamental flux appears essentially as a leakage component [15, 19]. As a consequence, even with an axially-laminated rotor, which allows the highest coupling coefficient [8], $k_{p s}$ turns out to be less than 0.8 leading to a compromised torque performance for the machine $[10,15]$.

Remark 7: The secondary power expression (7) clearly indicates the slip energy recovery nature of the BDFRM's operation as the supply inverter only has to handle the amount of power proportional to $\left|\omega_{s}=-s \omega_{p}\right|$ i.e. the degree of slip $s$. In this respect, the BDFRM behaves as a double-fed induction machine and this characteristic is the reason that a fractionally rated inverter can be used for restricted speed changes above and below the synchronous speed $\left(\omega_{s y n}=\omega_{p} / p_{r}\right)$ when $s$ is small $^{7}$. If the secondary was fed with the line frequency then the BDFRM would rotate at $2 \omega_{\text {syn }}$ (i.e. at the speed of a conventional $2 p_{r}$-pole synchronous machine) and the two windings would then evenly contribute to the machine's power output $\left(P_{p}=P_{s}\right)$. Therefore, from a real power perspective the inverter obviously needs to be rated at most to handle $50 \%$ (plus losses) of the machine's real power rating. Note that the inverter will need a larger rating if some of the flux producing current is handled by the secondary.

${ }^{6}$ Note that the maximum secondary power factor angle is not $\pi / 2$ i.e. the corresponding $d$-axis current is not zero as the control frame is primary (not secondary) flux oriented.

${ }^{7}$ This property makes the BDFRM an ideal brushless candidate for pumptype applications where the speed range required is typically $2: 1$ or less [2]. 
Remark 8: If $\omega_{s n}=s=0$ then DC is being fed to the secondary and the BDFRM is operating as a classical field controlled $2 p_{r}$-pole synchronous turbo-machine with $\alpha_{s}$ effectively becoming the torque angle [4]. The inverter only compensates for the secondary copper losses under this condition, as follows from (7). Supplying the primary from another converter, then a brushless variable speed synchronous machine suitable for high speed field weakened applications would be realised.

Remark 9: If $\omega_{s n}<0$ (which means the opposite phase sequence to the primary) then the power being taken from the grid by the primary is passing through the machine to be returned back to the supply via the secondary, suffering losses on its way. In this inefficient 'power circulating' operating region of the machine, a fully regenerative and appropriately rated inverter would be required for sustained operation. However, if this mode is only used for starting (the machine is at standstill for $s=-\omega_{s n}=1$ ) then resistive dumping would be quite sufficient. A more practical, more economical, and for the inverter less stressful solution is replace the inverter with external resistors and start the BDFRM as an induction machine [13].

Remark 10: The $i_{s d}$ dependent component terms in the copper loss expression (12) illustrate the possibility of controlling the amount of copper losses in the machine in much the same manner as the secondary reactive power $\left(Q_{s}\right)$ for a given torque output, i.e via $i_{s d}$. However, unlike the primary reactive power $\left(Q_{p}\right)$ case, the control of copper losses (and $Q_{s}$ ) is not decoupled from torque, due to the presence of the $i_{s q}$ component in (12). It is easy to show from (12) and its rearranged normalised form in Table I that the minimum copper losses (MCL) are achieved if: ${ }^{8}$

$$
i_{s d_{m c l}}=\frac{\lambda_{p} L_{p s}}{L_{p s}^{2}+\frac{R_{s}}{R_{p}} L_{p}^{2}} \Leftrightarrow \alpha_{s_{m c l}}=\tan ^{-1} \frac{r_{p}+r_{s} \zeta^{2}}{2 r_{p}} T_{n}
$$

where $\zeta=L_{p} / L_{p s}$ (see Appendix A). Therefore, in order to minimise $i_{s d}$ and hence maximise torque per inverter ampere under MCL conditions one needs to increase $R_{s} / R_{p}$ ratio which, given (20) of Appendix C, translates to: $n_{s} \geq n_{p}$, $A_{s} \leq A_{p}$ and $p<q$. It should be noted that the observation about the windings turns per pole relationship for improved torque per ampere performance $\left(n_{s} / n_{p} \geq 1\right)$ is consistent with that made in Remark 4.

Remark 11: Another important comment about the MCL control strategy is that, unlike all the others considered previously, it is the only one affected by the windings pole-numbers. This dependence is introduced via the stator resistances (see (12) and (15)) and it means that it does matter which winding is a power winding and which one has a control function. Issues related to this are the subject of the subsequent sections.

\section{CONTROL PROPERTIES AND TRADE-OFFS}

The following analysis shall assume that the BDFRM windings have the same number of effective turns per pole $\left(n_{s}=\right.$ $\left.n_{p}\right)$ i.e. $L_{p}=L_{s}$ and the same gauge copper wire i.e. the same cross section $\left(A_{s}=A_{p}\right)$. It will be based on the machine's normalised expressions in Table I of Appendix B. A

\footnotetext{
${ }^{8}$ Similar results can be obtained for a double excited slip ring induction ma-
} chine [20].

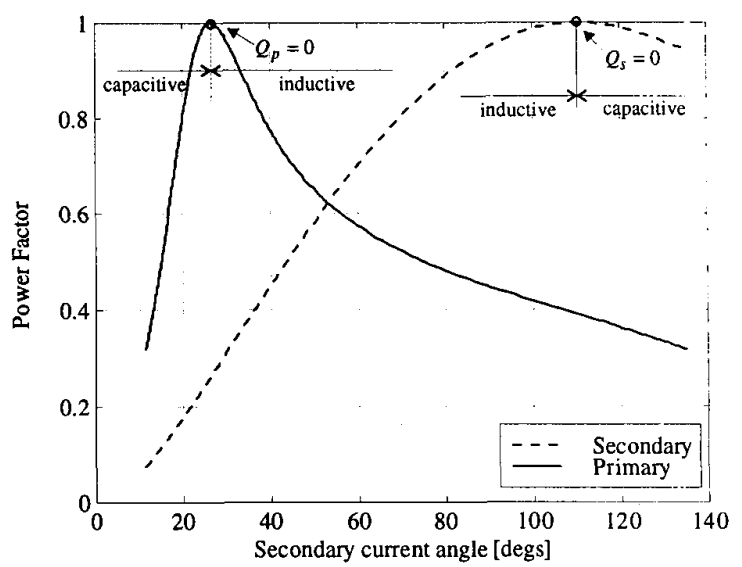

Fig. 3. Winding power factors for $T_{n}=1$

6/2-pole machine with a 4-pole rotor shall be considered assuming $k_{p s}=1 / \zeta=7 / 9$ (equivalent to a typical Syncrel rotor saliency ratio of $8[10])$.

\section{A. Constant torque operation}

Fig. 3 demonstrates the machine's ability to control primary (and secondary) power factor from leading to lagging via the secondary current angle. Notice that for $\alpha_{s}<\alpha_{s_{u p p l}}\left(\approx 26^{\circ}\right.$ under the conditions assumed) both power factors decrease towards zero as the primary winding is generating large amounts of reactive power into the grid, this being taken by the secondary winding from the inverter. It can be seen in Fig. 4 that in order to maintain the constant flux operation of the machine in this region the $\alpha_{p}$ angle increases to counteract the flux increasing effect of decreasing $\alpha_{s}$ angles. If $\alpha_{s}>\alpha_{s_{u p p}}$ then the primary power factor again deteriorates but with the primary now absorbing reactive power from the mains supply to flux the machine. The secondary power factor consequently improves reaching its maximum at $\alpha_{s_{u s p}} \approx 110^{\circ}$ when the primary winding becomes fully responsible for the flux production and the $\alpha_{p}$ values are small as shown in Fig. 4.

The impact of secondary current angle variations and winding pole-numbers on the machine's copper losses can be understood from Fig. 5. It can be seen that for the $p>q$ pole-pair combination (dashed curve) the MCL current angle is smaller than in $p<q$ case (solid line) indicating a higher $d$-axis secondary current $\left(i_{s d n}\right)$ and inverter current loading in the first case. This means that the torque per ampere is better under $p<q$ condition which conforms with Remark 10. At lower $\alpha_{s}$ angles both curves show a sharp increase of losses due to dominant secondary currents. The losses, as expected, are higher when $p<q$ since $R_{s}>R_{p}$ and $i_{p}<<i_{s}$. The situation is quite opposite at larger $\alpha_{s}$ values and small $i_{s}$, when the primary winding losses predominate. This trend is also evident from the third (dashed-dotted) characteristic in the same figure.

\section{B. Optimal control aspects at various torque levels}

The plots of (13), (14) and (15), for maximum power factor and minimum copper loss strategies implemented in a torque controller, are presented in Fig. 6 . The fact that the USPF sec- 


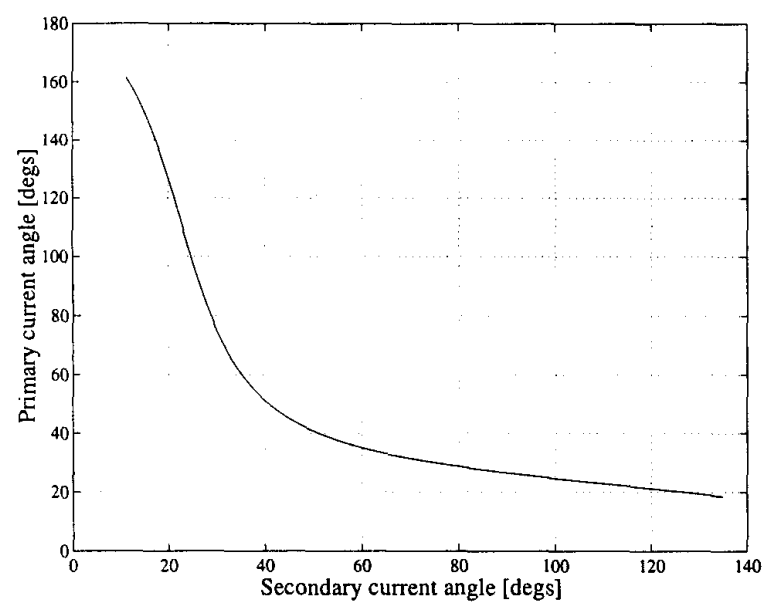

Fig. 4. Current angle relationship at $T_{n}=1$

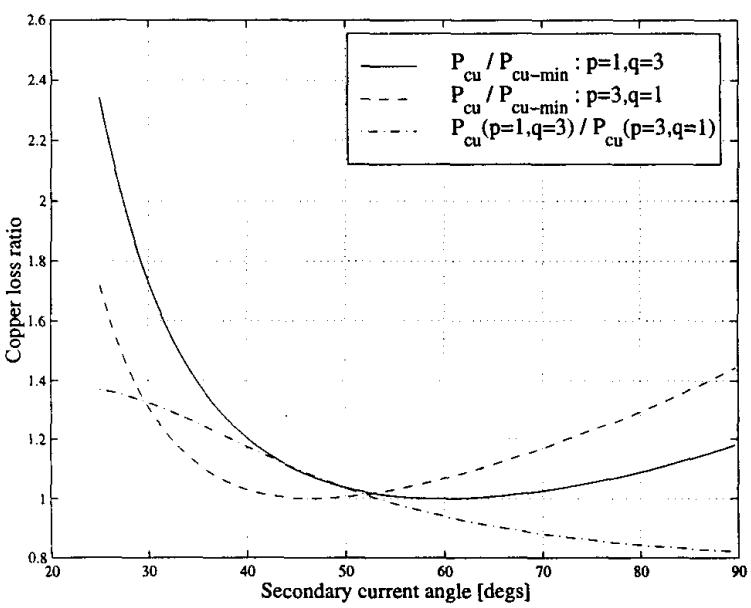

Fig. 5. Effects of winding pole-number combinations on copper losses at 1-pu torque

ondary current has a demagnetising effect (as $\alpha_{s_{u s p f}}>\pi / 2$ i.e. $i_{s d_{u s p f}}<0$ ) means that a significant extra $d$-axis current is needed in the primary to preserve constant flux in the machine. As a result, the USPF $i_{p n}$ is about 2.5 its UPPF value (Fig. 7) under 1-pu torque, the ratio being even higher at lower torques when most of the USPF current is flux producing (note the virtually flat curve in this torque range) and the UPPF current (a coupled $i_{s q n}$ as $\alpha_{p_{u p p}}=\pi / 2$ and $i_{p d n}=0$ ) is small. Note from Fig. 6 that the UPPF angles are the smallest of all as the secondary current is then entirely responsible for the machine magnetization and its $d$-axis component is at its largest.

From Fig. 6 one can also notice the higher values for the minimum copper loss (MCL) angles at any torque when $p<$ $q$. This indicates the lower secondary current magnitudes (at least 20\%) compared to the $p>q$ case as shown in Fig. 8 . Therefore, as far as the torque per inverter ampere strategy is concerned, the use of a high pole winding for control purposes is a preferable option as a smaller inverter is then needed.

Along the line of the previous discussion, it would be in-

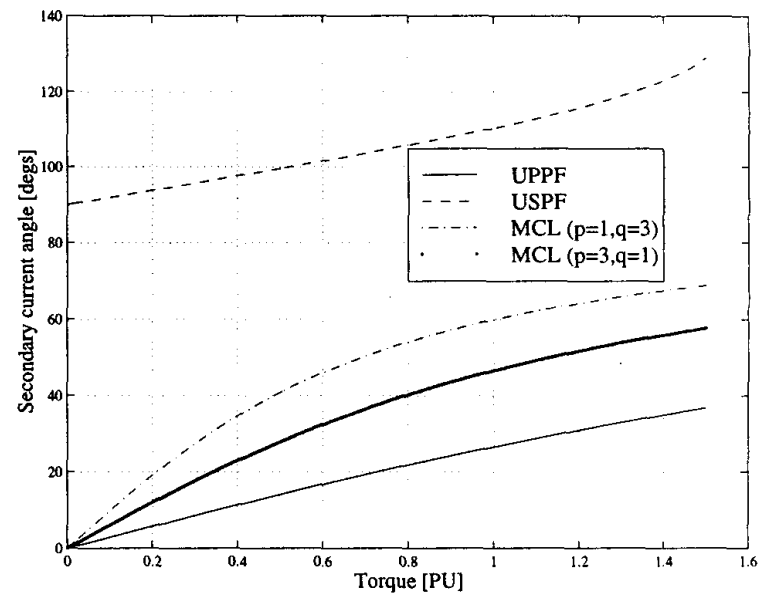

Fig. 6. Optimal control angles at various torque levels

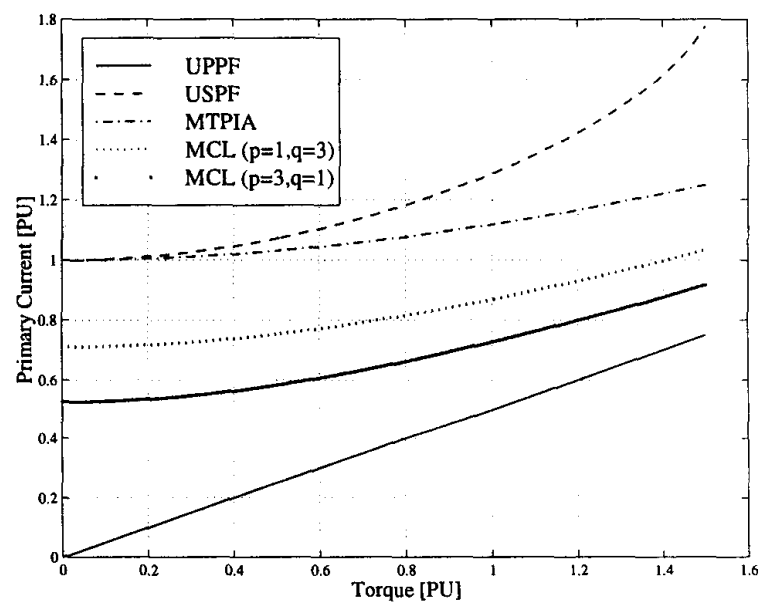

Fig. 7. Primary winding currents under optimal conditions

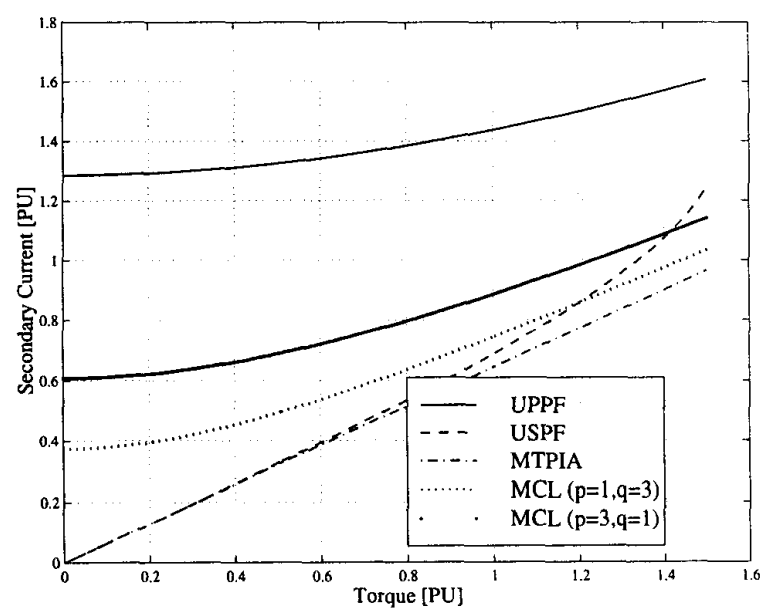

Fig. 8. Secondary winding currents for considered control methodologies 


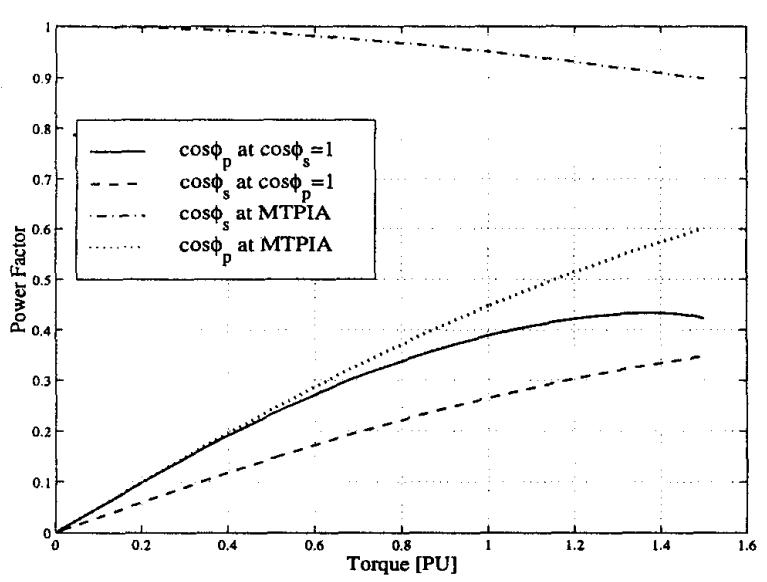

Fig. 9. Power factors for different control strategies

teresting to see what influence power factor control has on the inverter size required for a drive system. If the UPPF is desired, then the values of $\alpha_{s}$ are low and the secondary power factor is poor (Figs. 3 and 9) indicating an inefficient use of the inverter. Therefore, its current rating would have to be higher for a given output torque as illustrated by the top characteristic in Fig. 8 .

The situation is diametrically opposite under the USPF conditions when the $\alpha_{s}$ angles are above $\pi / 2$ and the secondary carries less reactive current. One can see in Fig. 8 that $i_{s n_{u s p f}} \approx 0.5 i_{s n_{u p p f}}$ at $T_{n}=1$ (and less at lower torque values) meaning that at least a half rated inverter can be used in this case. However, the primary power factor is then a compromise and its maximum value is 0.4 approximately (Fig. 9).

If the machine is operated at the maximum torque per secondary ampere (MTPSA), the primary power factor can be slightly improved (Fig. 9) relative to the USPF value and the inverter current rating further reduced (Fig. 8) but at the cost of moving away from the optimum inverter VA i.e. USPF point (Fig. 9).

The inverter current loading under MCL conditions for $p<$ $q$ is quite close to an optimum, but only around unity or higher pu torques (Fig. 8). The corresponding primary power factor is better than the MTPSA value but is still very low (less than 0.6 at 1-pu torque) as illustrated in Fig. 10. As expected, the secondary power factor is compromised relative to the MTPSA strategy (especially at lower torques) its value being 0.7 at unity torque as compared to approximately 0.95 under MTPSA conditions.

Another important aspect of this analysis is to examine the impact of the two pole-number combinations of the windings on the power factor performance of the machine with minimum copper losses. From Fig. 10 it is evident that the secondary power factor increases with torque for $p<q$ (dashed curve), as opposed to $p>q$ (dashed-dotted line). This results from the greater $\alpha_{s}$ angles and lower magnetising currents of the secondary winding in the first case (Fig. 6). The situation is opposite for primary power factor, due to the counteracting effect of the primary current angles required to maintain constant flux operation of the machine. This means that $\alpha_{p}$ decreases as $\alpha_{s}$ angles increase as shown in Fig. 4. As a consequence, for a primary power factor control strategy a multipole power

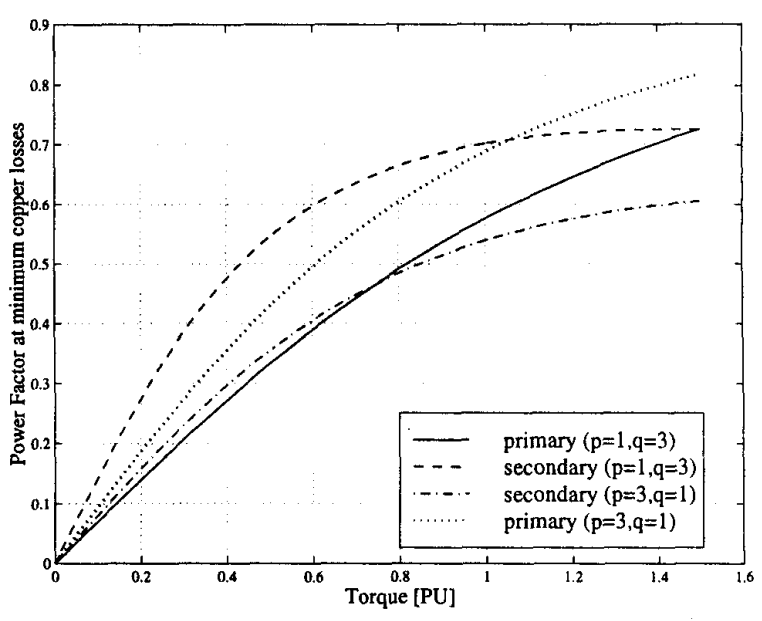

Fig. 10. Power factor performance with minimum copper losses

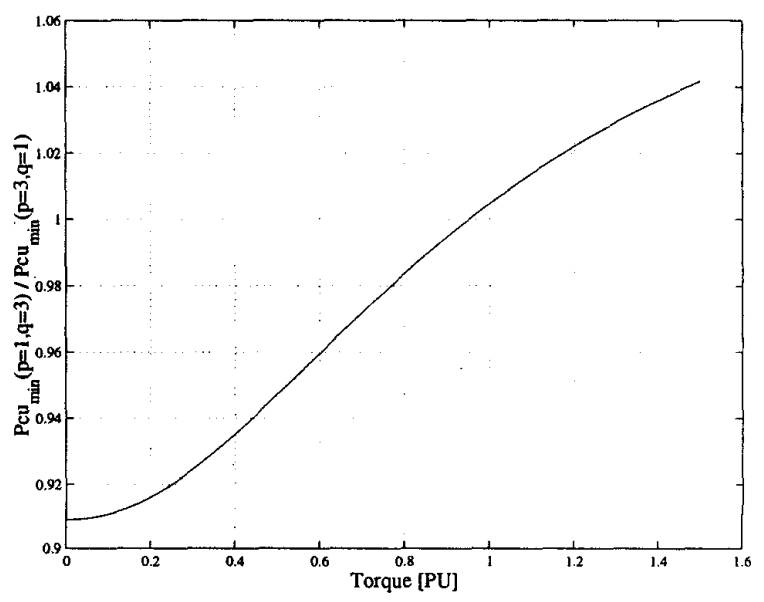

Fig. 11. Influence of winding pole-numbers on minimum copper losses

winding and a 2-pole control winding is a better solution.

From a viewpoint of minimum copper losses in the machine, the $p<q$ case appears to allow lower losses in the torque range up to about 0.95 -pu according to Fig. 11. At higher torque values, $p>q$ seems to be more efficient. The differences in losses are more pronounced at very low torques (less than 10\%), and hence the overall efficiency may be affected more significantly, as it has a tendency to decrease at small output powers. At mid range and higher torques, however, these variations are minor (only a few percent), and their impact on the machine efficiency is virtually negligible. Therefore, with respect to efficiency, unless the machine is to be used at low powers relative to its rating, it is irrelevant which winding is grid connected and which is inverter fed.

The final part of this paper is a comparative study of the control strategies in terms of torque per secondary ampere (TPSA) performance. The plots in Fig. 12 represent the TPSA normalised to the optimum MTPSA value (corresponding to $\alpha_{s}=\pi / 2$ ). The results in this figure fully match those in Fig. 


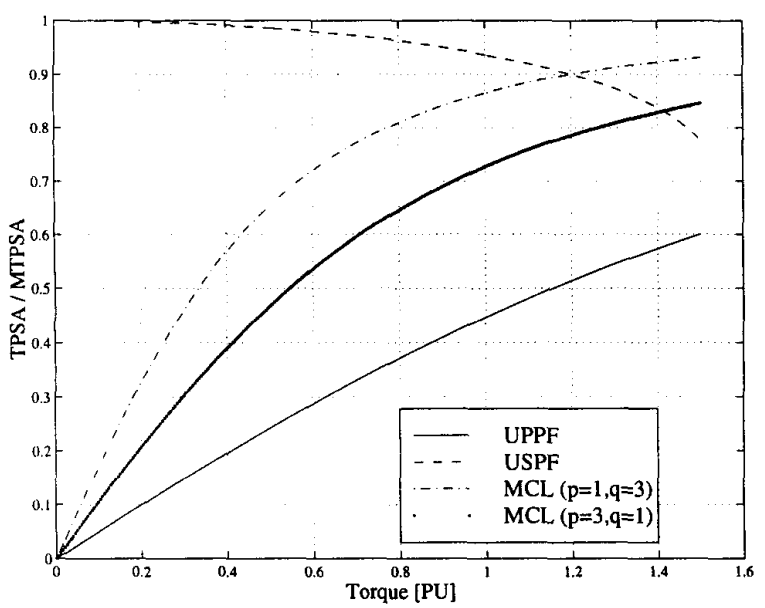

Fig. 12. Torque per secondary ampere at optimum power factors and minimum copper losses

8. After MTPSA strategy, the TPSA is the highest under USPF conditions, and it is the lowest at UPPF, when the inverter current loading is a maximum (as follows from Fig. 8). Fig. 12 yet again confirms the previously made conjecture of TPSA values being higher in the case of a machine with 2-pole primary and 6-pole secondary windings and minimum copper losses.

\section{CONCLUSIONS}

This paper has performed a comprehensive comparative analysis of the BDFRM's control properties and has developed a set of useful expressions suitable for implementation of various optimal control strategies for the machine. Aspects of power factor regulation and effects of winding pole-numbers on the machine performance have been particularly emphasised. The main conclusions/observations that can be made from the presented results are:

- The inverter current rating would have to be increased approximately twice for unity primary power factor (UPPF) control at the same torque output, as compared to the other strategies considered.

- Under the UPPF conditions the secondary power factor is less than 0.3 , indicating at least three times greater inverter $\mathrm{kVA}$ requirement for a given power output of a lossless machine. At the unity secondary power factor (USPF) and the minimum inverter kVA, the primary power factor is barely 0.4 .

- The maximum torque per secondary ampere and the USPF operating characteristics are quite close, especially at lower torques where they virtually coincide.

- All the performance parameters (except for the primary power factor), under minimum copper losses conditions, generally improve with a two-pole power winding (grid connected primary) and a multipole control winding (inverter-fed secondary). The primary power factor is better for the opposite situation (i.e. $p>q$ ).

- The above optimum pole-pair relationship $(p<q)$ allows lower copper losses and higher efficiency in the normal torque range, and particularly at smaller torque values.

Much of the material in the paper is an extract of the authors recent research $[15,21]$, and is not easily accessible in the lit-

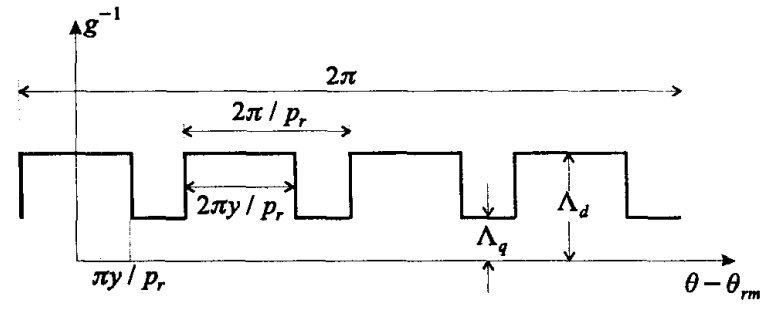

Fig. 13. Inverse air-gap function for a 4-pole reluctance rotor used for in ductance determination $(\theta \triangleq$ some mechanical angle around the stator circumference; $\theta_{r m} \triangleq$ the mechanical angle of the rotor high permeance axis)

erature. This work should make a significant contribution to fundamental knowledge and general understanding of the BDFRM operation and its optimal control. The consideration of the above performance/inverter rating trade-offs is important as in the long run the success or failure of this interesting and unusual machine will be likely decided by whether the compromise between a larger machine for a given output torque, and a smaller size of the inverter, lead to a lower system cost in the target applications.

The development of a 6/2-pole axially-laminated prototype and a controller is in its final stage at the University of Newcastle, Australia. The results of this work will be soon available and will be published in our future papers.

\section{APPENDIX}

\section{INDUCTANCE DEFINITIONS}

The BDFRM's inductance relationships listed below have been developed [15] using the method of winding functions [14] with the air-gap parameters as in Fig. 13. The expressions derived are, however, more general than those in [14] as they assume the nonzero permeance i.e. finite air-gap width along the rotor $q$-axis.

$$
\begin{gathered}
\text { Primary: } L_{p}=\frac{3}{2} \mu_{0} n_{p}^{2} m \pi r l+L_{l_{p}} \\
\text { Secondary: } L_{s}=\frac{3}{2} \mu_{0} n_{s}^{2} m \pi r l+L_{l_{s}} \\
\text { Mutual: } L_{p s}=\frac{3}{4} \mu_{0} n_{p} n_{s} n \pi r l \\
\zeta=\frac{L_{p}}{L_{p s}} \approx \frac{n_{p}}{n_{s}} \frac{2 m}{n} \\
m=y \Lambda_{d}+(1-y) \Lambda_{q} \\
n=\frac{2}{\pi}\left(\Lambda_{d}-\Lambda_{q}\right) \sin \pi y
\end{gathered}
$$

where:

$$
\begin{aligned}
n_{p, s} & \triangleq \text { effective turns/pole/phase of the windings } \\
L_{l_{p, s}} & \triangleq \text { leakage inductances of the windings } \\
r, l & \triangleq \text { machine mean radius and axial length } \\
1 / \Lambda_{d} & \triangleq \text { minimum air-gap width } \\
1 / \Lambda_{q} & \triangleq \text { maximum air-gap width } \\
y & \triangleq \text { pole-arc/pole-pitch ratio of the rotor (Fig. 13) }
\end{aligned}
$$

\section{NORMALISATIONS}

In order to make machine performance expressions independent of its parameters a set of normalisations corresponding to the arbitrary chosen $\alpha_{p}=\alpha_{s}=\pi / 4$ condition has been 
TABLE I

NORMALISED CONTROL FORM EXPRESSIONS AND BASE QUANTITIES

\begin{tabular}{|c|}
\hline Primary quantities \\
\hline$i_{p n}=\frac{\sin \alpha_{s}}{\sin \left(\alpha_{p}+\alpha_{s}\right)}=\frac{\sqrt{\left(2 \tan \alpha_{s}-T_{n}\right)^{2}+T_{n}^{2} \tan ^{2} \alpha_{s}}}{2 \tan \alpha_{s}}$ \\
\hline $\left.\begin{array}{c}\lambda_{p n}=i_{p d n}+\frac{1}{\zeta} i_{s d n}=1 \\
i_{p q n}-\frac{1}{\zeta} i_{s q n}=0\end{array}\right\} \Leftrightarrow \tan \alpha_{p}=\frac{T_{n} \tan \alpha_{s}}{2 \tan \alpha_{s}-T_{n}}$ \\
\hline$Q_{p n}=1-\frac{i_{s d_{n}}}{\zeta}=1-\frac{T_{n}}{2 \tan \alpha_{s}}$ \\
\hline $\cos \phi_{p}=\frac{P_{p n}}{\sqrt{P_{p n}^{2}+Q_{p n}^{2}}}=\frac{}{\sqrt{\left(T_{n}^{2}+4\right) \tan ^{2} \alpha_{s}-4 T_{n} \tan \alpha_{s}+T_{n}^{2}}}$ \\
\hline Secondary quantities \\
\hline$i_{s n}=\frac{\zeta \sin \alpha_{p}}{\sin \left(\alpha_{p}+\alpha_{s}\right)}=\frac{\zeta T_{n} \sqrt{1+\tan ^{2} \alpha_{s}}}{2 \tan \alpha_{s}}$ \\
\hline$P_{s n}=\frac{\omega_{s n}}{2} T_{n}=\omega_{s n} P_{p n}=-s P_{p n}$ \\
\hline$Q_{s n}=\frac{\omega_{s n} T_{n}}{4 \tan ^{2} \alpha_{s}}\left[T_{n}\left(\frac{1}{k_{p s}^{2}}-1\right)\left(\tan ^{2} \alpha_{s}+1\right)+2 \tan \alpha_{s}\right.$ \\
\hline$\phi_{s}=\tan ^{-1} \frac{\mathcal{Q}_{s n}}{P_{s n}}=\tan ^{-1} \frac{T_{n}\left(1 / k_{p s}^{2}-1\right)\left(\tan ^{2} \alpha_{s}+1\right)+2 \tan \alpha_{s}}{2 \tan ^{2} \alpha_{s}}$ \\
\hline Torque, power output and copper losses \\
\hline$T_{n}=\frac{2}{\zeta} i_{s q n}=\frac{2}{\zeta} i_{s n} \sin \alpha_{s}$ \\
\hline$P_{n}=P_{p n}+P_{s n}=\frac{1+\omega_{s n}}{2} T_{n}=\omega_{r n} T_{n}$ \\
\hline$P_{c u n}=\frac{4 r_{p} \tan \alpha_{s}\left(\tan \alpha_{s}-T_{n}\right)+T_{n}^{2}\left(r_{p}+r_{s} \zeta^{2}\right)\left(\tan ^{2} \alpha_{s}+1\right)}{4 \tan ^{2} \alpha_{s}}$ \\
\hline$P_{c u n_{\min }}=P_{c u n}\left(\alpha_{s_{m c l}}\right)=\frac{\left(r_{p}+r_{s} \zeta^{2}\right)^{2} T_{n}^{2}+4 r_{p} r_{s} \zeta^{2}}{4\left(r_{p}+r_{s} \zeta^{2}\right)}$ \\
\hline Base values \\
\hline Torque \& power: $T_{B}=\frac{3}{4} p_{r} \frac{\bar{\lambda}_{p}^{2}}{L_{p}}$ \\
\hline Current \& flux : $i_{B}=\lambda_{B} / L_{p}$ \\
\hline
\end{tabular}

developed taking the grid voltage and frequency as bases (see Table I) [9].

\section{STATOR RESISTANCE EXPRESSION}

Consider a single full-pitch turn of a generic $P$-pole sinusoidally distributed winding. The total length and resistance of the turn are:-

$$
\begin{aligned}
l_{t} & =\text { length in slots }+ \text { end turn length }=2 l+\frac{2 \pi d}{P} \\
R_{t} & =\frac{\rho_{c u} l_{t}}{A}=\frac{2 \rho_{c u}}{A}\left(l+\frac{\pi d}{P}\right)=\frac{2 \rho_{c u} d}{A}\left(\beta+\frac{\pi}{P}\right)
\end{aligned}
$$

where:

$$
\begin{aligned}
l & \triangleq \text { length of the machine stack } \\
d & \triangleq \text { diameter from the centre of the stator slots } \\
\rho_{c u} & \triangleq \text { resistivity of conductive material (copper) } \\
A & \triangleq \text { cross-sectional area of the wire used } \\
\beta & =l / d \triangleq \text { machine's aspect ratio }
\end{aligned}
$$

If the winding has $n$ effective turns per pole and phase then its total phase resistance is:-

$$
R=P \cdot n R_{t}=\frac{2 \rho_{c u} d}{A} n(P \beta+\pi)
$$

Applying this to the BDFRM windings and assuming $\beta \approx 0.5$ for convenience (a NEMA180 frame), then the resistances of the $2 q$-pole secondary and $2 p$-pole primary winding can be related as follows:-

$$
\frac{R_{s}}{R_{p}}=\frac{n_{s}}{n_{p}} \cdot \frac{A_{p}}{A_{s}} \cdot \frac{q+\pi}{p+\pi}
$$

\section{REFERENCES}

[1] Y. Liao, "Design of a brushless doubly-fed induction motor for adjustable speed drive applications," Proceedings of the IAS Annual Meeting, pp. 850-855, San Diego, California, October 1996.

[2] B.Gorti, D.Zhou, R.Spé, G.Alexander, and A.Wallace, "Development of a brushless doubly-fed machine for a limited speed pump drive in a waste water treatment plant," Proc. of the IEEE-IAS Annual Meeting, pp. 523529, Denver,Colorado,October 1994.

[3] L. Xu and Y. Tang, "A novel wind-power generating system using field orientation controlled doubly-excited brushless reluctance machine," Proc. of the IEEE IAS Annual Meeting, Houston, Texas, October 1992.

[4] O.Ojo and Z.Wu, "Synchronous operation of a dual-winding reluctance generator," IEEE Transactions on Energy Conversion, vol. 12, pp. 357362, December 1997.

[5] F.Saifkhani and A.K.Wallace, "A linear brushless doubly-fed machine drive for traction applications," Proc. of the European Power Electronics Conference, pp. 344-348, Brighton,UK,September 1993.

[6] A.K.Wallace, R.Spee, and G.C.Alexander, "Adjustable speed drive and variable speed generation systems with reduced power converter requirements," Proc. of the International Symposium on Industrial Electronics (ISIE), pp. 503-508, 1993.

[7] R.Li, R.Spee, A.K.Wallace, and G.C.Alexander, "Synchronous drive performance of brushless doubly-fed motors," IEEE Transactions on Indus try Applications, vol. 30, pp. 963-970, July/August 1994.

[8] L.Xu and F.Wang, "Comparative study of magnetic coupling for a doubly fed brushless machine with reluctance and cage rotors," IEEE-IAS Annual Meeting, pp. 326-332, New Orleans, Louisiana,October 1997

[9] R.Betz and M.Jovanovic, "Control aspects of brushless doubly fed reluctance machines," Proc. of the European Power Electronics Conference. Lausanne,Switzerland,September 1999.

[10] R.Betz and M.Jovanovic, "The brushless doubly fed reluctance machine and the synchronous reluctance machine - a comparison," Proc. of the IEEE-IAS Annual Meeting, Phoenix, Arizona,October 1999.

[11] M.S.Boger, A.K.Wallace, R.Spee, and R.Li, "General pole number model of the brushless doubly-fed machine," IEEE Transactions on Industry Applications, vol. 31, pp. 1022-1028, Sept/Oct 1995.

[12] D.Zhou, R.Spee, and G.C.Alexander, "Experimental evaluation of a rotor flux oriented control algorithm for brushless doubly-fed machines," IEEE Transactions on Power Electronics, vol. 12, pp. 72-78, January 1997.

[13] L. Xu, L. Zhen, and E. Kim, "Field-orientation control of a doubly excited brushless reluctance machine," IEEE Transactions on Industry Applications, vol. 34, pp. 148-155, Jan/Feb 1998.

[14] F. Liang, L. Xu, and T. Lipo, "D-q analysis of a variable speed doubly AC excited reluctance motor," Electric Machines and Power Systems, vol. 19, pp. 125-138, March 1991.

[15] R. Betz and M. Jovanovic,. "Introduction to brushless doubly fed reluctance machines - the basic equations," tech. rep., Dept. Elec. Energy Conversion, Aalborg University, Denmark, Dec. 1998. Available at www.ee.newcastle.edu.au/users/staff/reb/Betz.html.

[16] B.Hopfensperger, D.J.Atkinson, and R.A.Lakin, "Stator flux oriented control of a cascaded doubly-fed induction machine," IEE Proc. - Electr. Power Appl., vol. 146, pp. 597-605, November 1999.

[17] Y. Liao and C. Sun, "A novel position sensorless control scheme for doubly fed reluctance motor drives," IEEE Transactions on Industry Applications, vol. 30, pp. 1210-1218, Sept/Oct 1994.

[18] L.Xu and W.Cheng, "Torque and reactive power control of a doubly fed induction machine by position sensorless scheme," IEEE Transactions on Industry Applications, vol. 31, pp. 636-642, May/June 1995.

[19] Y. Liao, L. Xu, and L. Zhen, "Design of a doubly-fed reluctance motor for adjustable speed drives," IEEE Transactions on Industry Applications, vol. 32, pp. 1195-1203, Sept/Oct 1996.

[20] Y.Tang and L.Xu, "A flexible active and reactive power control strategy for a variable speed constant frequency generating system," IEEE Transactions on power electronics, vol. 10, pp. 472-478, July 1995

[21] R. Betz and M. Jovanović, "Comparison of the brushless doubly fed reluctance machine and the synchronous reluctance machine." tech. rep., Dept. of Elec. and Comp. Eng., University of Newcastle, Australia, 1999. 